\title{
BV Estimates Fail for Most Quasilinear Hyperbolic Systems in Dimensions Greater Than One
}

\author{
Jeffrey Rauch * \\ University of Michigan, Ann Arbor, USA and \\ Centre de Mathématiques Appliquées, Ecole Polytechnique, F-91128 Palaiseau, France
}

\begin{abstract}
We show that for most non-scalar systems of conservation laws in dimension greater than one, one does not have BV estimates of the form

$$
\begin{gathered}
\|\nabla u(\bar{t})\|_{\text {T.v. }} \leqq F\left(\|\nabla u(0)\|_{\text {T.v. }}\right), \\
F \in C(\mathbb{R}), \quad F(0)=0, \quad F \text { Lipshitzean at } 0,
\end{gathered}
$$

even for smooth solutions close to constants. Analogous estimates for $L^{p}$ norms

$$
\|u(\bar{t})-\bar{u}\|_{L^{p}} \leqq F\left(\|u(0)-\bar{u}\|_{L^{p}}\right), \quad p \neq 2
$$
\end{abstract}

with $F$ as above are also false. In one dimension such estimates are the backbone of the existing theory.

The assertions of the abstract are fairly direct consequences of the fact that, except for trivial cases, linear hyperbolic systems in dimension greater than one are not well posed in $L^{p}$ for $p \neq 2$. One might hope that the conservation laws are better behaved than the linear systems. For example, in one space dimension the solution operator $u(0) \rightarrow u(t), t>0$, maps $L^{\infty}$ to $\mathrm{BV}$, a smoothing property not shared by linear equations. It is the purpose of this note to dash such hopes. The analysis is made entirely within the framework of smooth solutions so that neither conservation form nor entropy conditions play a role.

The $k \times k$ systems for $u(t, x), t, x \in \mathbb{R} \times \mathbb{R}^{d}, u \in \mathbb{R}^{k}$ are assumed to have the form

$$
A_{0}(u) \partial_{t} u+\sum_{j=1}^{d} A_{j}(u) \partial_{j} u+B(u)=0,
$$

where $\partial_{j} \equiv \partial / \partial x_{j}$ and

$$
A_{j} \in C^{\infty}\left(\mathbb{R}^{k}: \operatorname{Hom}\left(\mathbb{R}^{k}\right)\right), \quad B \in C^{\infty}\left(\mathbb{R}^{k}: \mathbb{R}^{k}\right) .
$$

\footnotetext{
* Research partially supported by the National Science Foundation under grant MCS-8301061
} 
We assume that the system is symmetrizeable hyperbolic or strictly hyperbolic (see [5]) on a neighborhood of a vector $\bar{u} \in \mathbb{R}^{k}$, and $B(\bar{u})=0$. The hyperbolicity guarantees the existence of classical solutions, local in time.

Local Existence Theorem. There is an $s=s(d)>d / 2+1$ and $\varrho>0$ such that for all $\varphi \in C_{0}^{\infty}\left(\mathbb{R}^{d}\right),\|\varphi\|_{H^{s}\left(\mathbb{R}^{d}\right)}<\varrho$, there is a unique solution to (1) on $|t| \leqq T \equiv c /\|\varphi\|_{H^{s}}$ with $\left.u\right|_{t=0}=\bar{u}+\varphi, u-\bar{u} \in C_{(0)}^{\infty}\left([-T, T] \times \mathbb{R}^{d}\right)$. The map $\varphi \rightarrow u-\bar{u}$ is $C^{\infty}$ from $H^{s}\left(\mathbb{R}^{d}\right)$ to $C\left([-T, T]: H^{s}\left(\mathbb{R}^{d}\right)\right)$ with derivative calculated using the linearized equation.

This is a result dating from the work of Schauder in the thirties (see [3] for references and a classical proof). Modern proofs are presented in $[4 ; 5$ Chap. 2,6]. Our main result is the following theorem:

Theorem. If there are positive constants $c, \eta$ and $\bar{t}>0$ so that for all $\varphi \in C_{0}^{\infty}\left(\mathbb{R}^{d}\right)$ with $\|\varphi\|_{H^{s}\left(\mathbb{R}^{d}\right)}<\eta$ the solution of (1) with Cauchy data $\bar{u}+\varphi$ satisfies

$$
\int_{\mathbb{R}^{d}}\left|\nabla_{x} u(\bar{t}, x)\right| d x \leqq c \int_{\mathbb{R}^{d}}\left|\nabla_{x} u(0, x)\right| d x
$$

then for all $j, l$

$$
\left[A_{0}(\bar{u})^{-1} A_{j}(\bar{u}), A_{0}(\bar{u})^{-1} A_{l}(\bar{u})\right]=0,
$$

where $[$,$] denotes commutator.$

Thus, the algebraic identities (3) are necessary conditions for the existence of BV bounds.

Example 1. In the one-dimensional case, $d=1$, there is only one matrix $A_{0}^{-1} A_{1}$ and (3) is trivially satisfied.

Example 2. In the scalar case, $k=1$, the matrices $A_{0}^{-1} A_{j}$ are $1 \times 1$ and therefore commute.

Example 3. All the inviscid equations of compressible fluid dynamics violate (3), so there is no hope for BV estimates in these important cases. A simple case is twodimensional isentropic flow (see [3, p. 600] for the equations) where condition (3) is violated for all $u$. Increasing the number of dimensions, passing to the nonisentropic case, adding electromagnetic or relativistic effects, ... makes matters only worse.

Proof of Theorem. Consider Cauchy data $u_{\varepsilon}(0, \cdot)=\bar{u}+\varepsilon \varphi(\cdot), \varphi \in C_{0}^{\infty}\left(\mathbb{R}^{d}\right)$. Then $u_{\varepsilon}=\bar{u}+\varepsilon v+r_{\varepsilon}$, where $v$ is the solution of the linearized equation,

$$
\left[A_{0}(\bar{u}) \partial_{t}+\sum A_{j}(\bar{u}) \partial_{j}+B^{\prime}(\bar{u})\right] v=0,\left.\quad v\right|_{t=0}=\varphi,
$$

and for any $s>0$

$$
\sup _{0 \leqq t \leqq \bar{t}}\left\|r_{\varepsilon}(t)\right\|_{H^{s}\left(\mathbb{R}^{d}\right)}=O\left(\varepsilon^{2}\right)
$$

In particular, $\left\|\nabla_{x} r_{\varepsilon}(\bar{t})\right\|_{L^{2}\left(\mathbb{R}^{d}\right)}=O\left(\varepsilon^{2}\right)$. Using the finite speed of propagation for (1) we see that the $r_{\varepsilon}$ are supported in a fixed compact subset of $[-\bar{t}, \bar{t}] \times \mathbb{R}^{d}$ so $\left\|\nabla_{x} r(\bar{t})\right\|_{L^{1}\left(\mathbb{R}^{d}\right)}=O\left(\varepsilon^{2}\right)$. 
From (2) we have:

$$
c\left\|\nabla_{x} \varepsilon \varphi\right\|_{L^{1}} \geqq\left\|\nabla_{x} u_{\varepsilon}(\bar{t})\right\|_{L^{1}} \geqq \varepsilon\|v(\bar{t})\|_{L^{1}}-O\left(\varepsilon^{2}\right) .
$$

Dividing by $\varepsilon$ and letting $\varepsilon \rightarrow 0$ yields

$$
\left\|\nabla_{x} v(t)\right\|_{L^{1}\left(\mathbb{R}^{d}\right)} \leqq c\|\nabla \varphi\|_{L^{1}\left(\mathbb{R}^{d}\right)}, \quad \forall \varphi C C_{0}^{\infty} .
$$

Let $M(\xi)$ be the $\operatorname{Hom}\left(C^{k}\right)$ valued function

$$
M(\xi) \equiv \exp \left(\bar{t} A_{0}(\bar{u})^{-1}\left(-\sum_{j=1}^{d} A_{j}(\bar{u}) i \xi_{j}-B^{\prime}(\bar{u})\right)\right),
$$

and let $M_{\alpha \beta}$ be the components of the matrix $M$. In (5) consider $\varphi$ all of whose components are zero except the $\beta^{\text {th }}$ component and look at the gradient of the $\alpha^{\text {th }}$ component of $v$ to find that $\left(D \equiv\left(\partial_{1}, \ldots, \partial_{n}\right)\right)$

$$
\left\|D_{l} M_{\alpha \beta}(D) \chi\right\|_{L^{1}}<c\|\nabla \chi\|_{L^{1}}, \quad \forall \chi \subset C_{0}^{\infty}\left(\mathbb{R}^{n}\right), \quad 1 \leqq l \leqq n .
$$

Let $\hat{\psi} \equiv|\xi| \hat{\chi}$. Then

$$
\left\|\frac{D_{l}}{|D|} M_{\alpha \beta}(D) \psi\right\|_{L^{1}} \leqq c \sum_{j}\left\|\frac{D_{j}}{|D|} \psi\right\|_{L^{1}} .
$$

Now, the hyperbolicity of (1) shows that (5) is valid with $L^{1}$ replaced by $L^{2}$, with a new constant $c$. Interpolating, (5) is valid for $L^{p}, 1 \leqq p \leqq 2$ with a constant independent of $p$. Repeating the derivation of (6) we find that (6) is valid for $L^{p}$, $1 \leqq p \leqq 2$. Now for $1<p<\infty, D_{j} /|D|$ is a bounded operator on $L^{p}\left(\mathbb{R}^{n}\right)$ so

$$
\left\|\frac{D_{l}}{|D|} M_{\alpha \beta}(D) \psi\right\|_{L^{p}} \leqq c_{p}\|\psi\|_{L^{p}}
$$

This holds for all $\psi \in|D| C_{0}^{\infty}\left(\mathbb{R}^{n}\right)$ which is a dense subset of $L^{p}$, so $D_{l} M_{\alpha \beta}(D) /|D|$ is a bounded operator on $L^{p}\left(\mathbb{R}^{n}\right)$ for all $1<p \leqq 2$.

To show that $M(D)$ is itself bounded, multiply by $D_{l} /|D| \in \operatorname{Hom}\left(L^{p}\right)$ and sum on $l$.

We now appeal to the result of Brenner $[1,2]$ which states that (3) is a necessary and sufficient condition for $M$ to be an $L^{p}$ multiplier for some $1<p<\infty, p \neq 2$.

Remark. It is even easier to show that the commutation relation (3) is also a necessary condition for strong $L^{p}$ estimates for $p \neq 2$. Precisely, if there is a $p \neq 2$ and $F \in C(\mathbb{R})$, with $F(0)=0$ and $F$ Lipshitzean at 0 such that

$$
\|u(\bar{t})-\bar{u}\|_{L^{p}\left(\mathbb{R}^{d}\right)} \leqq F\left(\|u(0)-\bar{u}\|_{L^{p}\left(\mathbb{R}^{d}\right)}\right)
$$

for the solutions discussed above, then (3) must hold. The proof is exactly like the proof of Theorem 1 with the exception that one finds immediately, at the level of Eq. (5), that $M(D)$ is in $\operatorname{Hom}\left(L^{p}\right)$. Temple [7] calls the estimate (7) strong $L^{p}$ stability of the constant state $\bar{u}$. Looking at the interaction of weak nearly constant shocks, he has shown that such estimates are false for all $p \neq 1$. Thus for non-trivial systems of conservation laws in dimension greater than one, constant states are not strongly stable in $L^{p}$ for any $p$ ! 


\section{References}

1. Brenner, $\mathrm{Ph} .:$ The Cauchy problem for the symmetric hyperbolic systems in $L_{p}$. Math. Scand. 19, 27-37 (1966)

2. Brenner, Ph.: The Cauchy problem for systems in $L_{p}$ and $L_{p, \alpha}$. Ark. Mat. 11, 75-101 (1973)

3. Courant, R.: Methods of mathematical physics, Vol. II. New York: Interscience 1966

4. Lax, P.D.: Nonlinear hyperbolic systems. Stanford University Lecture Notes 1970

5. Majda, A.: Compressible fluid flow and systems of conservation laws in several space variables. Appl. Math. Series No. 53. Berlin, Heidelberg, New York: Springer 1984

6. Taylor, M.: Pseudodifferential operators. Princeton, NJ: Princeton University Press 1981

7. Temple, B.: Preprint

Communicated by L. Nirenberg

Received February 20, 1986; in revised form April 24, 1986 